\title{
CONSUMO ELÉCTRICO EN GRANJAS DE GANADO OVINO LECHERO DE CASTILLA Y LEÓN
}

\section{ELECTRICITY CONSUMPTION IN CASTILLA Y LEÓN DAIRY SHEEP FARMS}

\author{
Bodas, R.*; Tabernero de Paz, M.J.; Bartolomé, D.J.; Posado, R.; García, J.J.; Olmedo, S. \\ y Rodríguez, L.
}

Instituto Tecnológico Agrario. Subdirección de Investigación y Tecnología. Consejería de Agricultura y Ganadería. Junta de Castilla y León. Finca Zamadueñas. Valladolid. España. *bodrodra@itacyl.es

\author{
Palabras clave adicionales \\ Ahorro. Eficiencia. Energía. Ordeño.
}

\section{RESUMEN}

Para conocer las características del consumo de energía y agua de las explotaciones de ganado ovino lechero de Castilla y León se realizó una auditoría energética a 35 granjas. Se recogieron datos de censo y producción, equipos y horario de funcionamiento (ordeño, enfriamiento, bomba de agua, agua caliente...), maquinaria disponible y consumo de agua. Se calcularon los índices de utilización de la energía por oveja productora y por unidad de leche producida. Aunque existen diferencias en función del tamaño de las explotaciones, el consumo de agua caliente sanitaria se sitúa en 0,42 L por oveja productora al día o 0,29 L por cada litro de leche producido. El consumo medio de electricidad es de $2000 \mathrm{kWh}$ al mes, es decir, casi $50 \mathrm{kWh} /$ oveja y año u $84 \mathrm{kWh}$ por cada $1000 \mathrm{~L}$ de leche producida. El ordeño consume $34 \mathrm{kWh} /$ día (más del $40 \%$ del gasto eléctrico diario), seguido del enfriamiento de la leche (30\%) y el calentamiento del agua (12\%). Las explotaciones con variador de velocidad en la bomba de vacío presentaron un menor consumo de electricidad por oveja. Se ha estimado que el consumo total de electricidad representa el $2 \%$ del precio percibido por cada litro de leche vendido.

\section{SUMMARY}

An energy audit model was designed and data collected on 35 dairy sheep farms in Castilla y León were used to ascertain the nature of energy and water consumption of these farms. Data regarding census and production, equipment and hours of

\author{
AdDitiOnAL KEYWORDS \\ Efficiency. Energy. Milking. Saving.
}

operation (milking, cooling, water pump, hot water...), other available machinery and water consumption were studied. Rates of energy utilization per producing ewe and per unit of milk produced were calculated. Beyond the differences due to farm size, hot water consumption was at $0.42 \mathrm{~L}$ daily per producing ewe producing or 0.29 $L$ per litre of produced milk produced. The average electricity consumption was $2000 \mathrm{kWh} /$ month, nearly $50 \mathrm{kWh} / \mathrm{ewe}$ and year or $84 \mathrm{kWh} / 1000 \mathrm{~L}$ of produced milk. Milking consumes $34 \mathrm{kWh} /$ day (more than $40 \%$ of daily utility costs), followed by milk cooling (30\%) and water heating (12\%). Farms with variable speed vacuum pump had lower electricity consumption by sheep. It has been estimated that total electricity consumption accounts for $2 \%$ of milk price.

\section{INTRODUCCIÓN}

Castilla y León ocupa el primer lugar a nivel nacional en censo de ovino de leche (1,25 millones de ovejas) y en producción de leche de oveja (386 millones de litros) (MARM, 2011). En los últimos 10 años se ha producido un descenso en el número de explotaciones (de 15000 explotaciones de ovino en 1999 a 9000 en 2009; INE, 2002, 2012), acompañado de un incremento en el número de animales por explotación (de 311 en 1999 a 380 en 2009). Si bien los sistemas tradicionales de producción de ovino le- 
chero se basaban en el empleo de mano de obra familiar y razas autóctonas, los cambios sufridos en el sector han hecho que este evolucione, impulsando a los ganaderos a utilizar cruces con razas foráneas y a implementar sistemas de mecanización y automatización. En consecuencia se ha producido un incremento en la productividad por animal y un aumento en la competitividad de las explotaciones (la cantidad de leche de oveja producida en Castilla y León ha aumentado casi un $80 \%$ en los últimos 10 años, según los datos del Ministerio de Agricultura, Alimentación y Medio Ambiente). Estos cambios han permitido a los ganaderos suplir la carencia de mano de obra disponible y ofertar empleo en condiciones laborales y económicas equiparables a otros sectores, en zonas donde las alternativas a esta actividad son escasas.

Sin embargo, el proceso de intensificación y el incremento en la productividad han repercutido en un aumento del consumo energético y de agua de las granjas, que pueden comprometer la competitividad de estas explotaciones. No existen estudios en la bibliografía relativos al consumo energético en explotaciones de ganado ovino lechero. Como paso previo a una gestión más racional y sostenible de las explotaciones debe tratarse de hacer un uso responsable de la energía, optimizar su consumo, minimizar el consiguiente impacto ambiental y mantener la viabilidad de la explotación. El objetivo del estudio fue la recogida de datos para conocer las características del consumo de energía y agua de las explotaciones encuestadas.

\section{MATERIAL Y MÉTODOS}

Se diseñó un modelo de auditoría, estructurada en varios apartados destacando:

- Identificación de la explotación: actividad principal, producción de leche anual, otras producciones (leche, carne, lana...).

- Características de funcionamiento de los principales equipos: sala de ordeño (tipo, horario, $\mathrm{n}^{\circ}$ de plazas), tanque de frío (capacidad, compacto o externo, intercambiador de calor, preenfriamiento de la leche).

- Datos de la producción y explotación ganadera: censo de animales y raza, lote medio de ovejas en producción, animales de recría, superficie agraria trabajada, labores contratadas o externas.

- Consumos eléctricos: tipo de iluminación, potencia y elementos en la explotación (tanto interior como exterior); motores presentes y sus características (bomba de vacío, bomba de la leche, molinos, bomba de agua, cintas de alimentación, nodrizas...), termo eléctrico (potencia, capacidad, aislamiento) y cualquier otro equipo; datos de consumo de agua caliente (limpieza del equipo de ordeño y del tanque, temperatura de mantenimiento del agua).

- Maquinaria disponible: tractores (potencia, antigüedad, horas de uso, mantenimiento), características de otra maquinaria autopropulsada y otros vehículos.

- Consumo de agua: procedencia (traída, sondeo, pozo), contador y consumo anual.

Se realizaron un total de 35 auditorias energéticas a explotaciones de ganado ovino distribuidas por Castilla y León (León, Palencia, Valladolid y Zamora). Las auditorías se recogieron por escrito, cotejando los datos facilitados con las correspondientes facturas de los últimos 12 meses. Todas las granjas auditadas tenían un sistema de explotación intensivo. La mayoría de las explotaciones (el $66 \%$ ) tenían animales de raza Assaf, mientras que el resto explotaban otras razas en pureza (Lacaune mayoritariamente) o mezcladas con Assaf. La productividad y adaptación de esta raza explican el éxito de su implantación en España (Lavín et al., 1997; Rodríguez et al., 2003; Ugarte et al., 2001), lo cual ha contribuido de manera definitiva a una mejora de las explotaciones lecheras (Castañares, 2008).

Se han calculado los índices de utilización de la energía por oveja productora y por unidad de leche producida (Edens et al., 
2003; Ludington y Johnson, 2003; Murgia et al., 2008). Para los cálculos económicos se ha utilizado el precio de la electricidad (1 $\mathrm{kWh}=0,13$ euros) en el momento de la realización del estudio (enero de 2011), disponible en la página web del Ministerio de Industria, Energía y Turismo (www.minetur. gob.es).

Para analizar los resultados, las explotaciones encuestadas se clasificaron según su tamaño (pequeñas $\leq 600$ ovejas, medianas: $601-1199$ ovejas, grandes: $\geq 1200$ ovejas) y la raza explotada (Assaf u otras). Los resultados obtenidos fueron sometidos a un análisis de varianza de dos vías, con el tamaño de explotación y la raza explotada como fuentes de variación. Dado que la interacción no resultó significativa en ningún caso, no se muestra en las tablas. Las medias de los grupos de tamaño se compararon utilizando el test DMS. El análisis estadístico se realizó utilizando el modelo lineal general univariante de SPSS 16.0 para Windows (IBM Corp., Nueva York, EE. UU.).

\section{RESULTADOSYDISCUSIÓN}

El tipo medio de explotación obtenido de las encuestas realizadas en Castilla y León se correspondería con una granja que tiene $532 \pm 49,5$ animales en ordeño, una producción de $856 \pm 98,6 \mathrm{~L}$ al día y unas $70 \pm 13,1$ ha, que trabajaría con $2 \pm 0,2$ tractores, cuya edad media ronda los $15 \pm 2,0$ años, y que tienen una potencia media de $102 \pm 4,3 \mathrm{CV}$ y una media de $592 \pm 56,6$ horas trabajadas por vehículo al año.

El tamaño de la explotación $(\mathrm{p}<0,001)$, pero no la raza $(p>0,10)$ condicionan el número medio de ovejas en ordeño, así como la producción media de leche. En este sentido, las granjas de tamaño medio mostraron los valores más altos de producción de leche por oveja, y las granjas de tamaño pequeño los más bajos, mientras que las granjas grandes ocuparon una posición intermedia ( $\mathrm{p}<0,01$, tabla I). Sin embargo, no se observaron diferencias significativas entre las granjas medianas y grandes en la producción de leche por día (pese a que numéricamente fue un $14 \%$ superior en las grandes que en las medianas), parámetro que sí fue inferior para las explotaciones más pequeñas $(\mathrm{p}<0,01)$ con respecto a los otros dos grupos.

En una explotación de ovino lechero, el ordeño y tratamiento de la leche obtenida en el mismo (bombeo hacia el tanque, enfriado, etc.), son los responsables de la mayor parte de su consumo eléctrico. Por otro lado, el agua caliente empleada para la limpieza e higiene de todos los elementos utilizados se obtiene gracias a la utilización de termos eléctricos o calderas de combustión (gasoil, butano, propano). Por consiguiente, previo al análisis de los consumos medios en las

Tabla I. Características de las explotaciones en función de su tamaño y de la raza explotada. (Farm characteristics depending upon their size and breed raised).

\begin{tabular}{lcccccccc}
\hline & \multicolumn{3}{c}{ Tamaño } & \multicolumn{3}{c}{ Raza } \\
& $\begin{array}{c}\text { pequeñas } \\
(\mathrm{n}=12)\end{array}$ & $\begin{array}{c}\text { medianas } \\
(\mathrm{n}=14)\end{array}$ & $\begin{array}{c}\text { grandes } \\
(\mathrm{n}=9)\end{array}$ & $\mathrm{p}$ & $\begin{array}{c}\text { Assaf } \\
(\mathrm{n}=23)\end{array}$ & $\begin{array}{c}\text { Otras } \\
(\mathrm{n}=12)\end{array}$ & $\mathrm{p}$ & der \\
\hline № animales & $442^{\mathrm{a}}$ & $884^{\mathrm{b}}$ & $1650^{\mathrm{c}}$ & $* * *$ & 889 & 1022 & $\mathrm{~ns}$ & 232,2 \\
Ovejas en ordeño & $259^{\mathrm{a}}$ & $559^{\mathrm{b}}$ & $792^{\mathrm{c}}$ & $* * *$ & 528 & 518 & $\mathrm{~ns}$ & 198,0 \\
Producción leche/oveja (L) & $12^{\mathrm{a}}$ & $1,94^{\mathrm{b}}$ & $1,55^{\mathrm{ab}}$ & $*$ & 1,65 & 1,57 & $\mathrm{~ns}$ & 0,628 \\
Producción leche/día (L) & $314^{\mathrm{a}}$ & $1053^{\mathrm{b}}$ & $1202^{\mathrm{b}}$ & $* * *$ & 899 & 756 & $\mathrm{~ns}$ & 434,0 \\
\hline
\end{tabular}

ns $=p>0,05 ;{ }^{*} p<0,05 ;{ }^{* * *} p<0,001 ;$ der $=$ desviación estándar residual. 


\section{BODAS ETAL.}

granjas, es conveniente conocer los parámetros relacionados tanto con la eficiencia del ordeño como con el consumo de agua caliente sanitaria (tabla I). El tiempo dedicado al ordeño de las ovejas depende tanto del número de animales a ordeñar como de las características de la sala de ordeño y la mano de obra disponible y se sitúa alrededor de los $40 \pm 3,8$ min por cada 100 ovejas. No obstante, la eficiencia en el ordeño depende del tamaño de la explotación. Así, el tiempo necesario para ordeñar 100 ovejas en las explotaciones pequeñas es en torno al doble $(\mathrm{p}<0,001)$ que en las medianas o pequeñas (entre las cuales no hay diferencias (tabla II). La capacidad media del tanque para todas las granjas fue de $2573 \mathrm{~L}$, presentando las explotaciones grandes y medianas una mayor capacidad de almacenamiento de leche que las más pequeñas $(p<0,01)$. No se observaron, en cambio, diferencias en cuanto a la relación entre la capacidad del tanque y la producción de leche por oveja o por día ( $p>0,10)$, situándose los valores medios en 5,2 $\pm 0,50$ litros de capacidad por oveja en producción y
3,4 $\pm 0,34$ L por litro de leche producido al día. Estos parámetros parecen estar relacionados, por una parte, con la frecuencia de retirada de la leche del tanque (bien sea por el servicio de recogida del comprador, o bien por el propio ganadero para llevarla a la cuba de cuajar y elaborar quesos). Sin embargo, por otra parte, hay que considerar que, a igualdad de potencia del tanque de frío, cuanto mayor sea la superficie del tanque en relación al volumen de leche producido, en menos tiempo se logrará bajar la temperatura de la leche.

En cuanto al consumo de agua caliente sanitaria, éste se sitúa en $0,42 \mathrm{~L}$ por cada oveja en producción de la explotación al día o 0,29 L por cada litro de leche producido. Obviamente, al aumentar la producción de leche diaria esta relación disminuirá, porque la cantidad de agua necesaria para la limpieza del circuito de ordeño será la misma. Estos consumos fueron inversamente proporcionales al tamaño de las explotaciones $(\mathrm{p}<0,05)$, es decir, a mayor tamaño de explotación, menor consumo relativo de agua. El consumo de agua caliente es mayor en tér-

Tabla II. Eficiencia de ordeño y de consumo de agua caliente sanitaria en las granjas de ganado ovino lechero de Castilla y León en función de su tamaño y de la raza explotada. (Milking efficiency and hot water consumption in dairy sheep farms of Castilla y León in terms of size and breed raised).

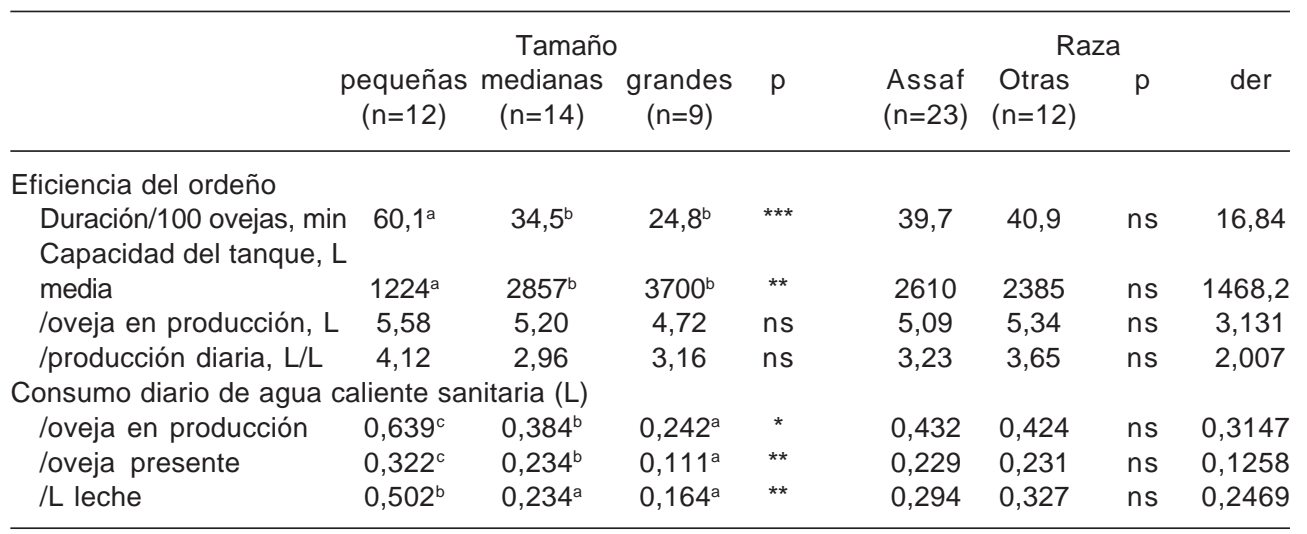

ns $=p>0,05 ;{ }^{*} p<0,05 ;{ }^{* *} p<0,01 ;{ }^{* * *} p<0,001 ;$ der= desviación estándar residual. 
minos absolutos en las explotaciones más grandes, pero éstas hacen un uso más eficiente en relación con la cantidad de leche producida o con el número de animales presentes. No se observaron, por el contrario, diferencias debidas al tipo de raza explotada $(\mathrm{p}>0,10)$.

En la tabla III se muestra el consumo medio de electricidad de las explotaciones, el cual se sitúa en algo menos de 2000 kWh al mes, lo que equivale a casi $50 \mathrm{kWh}$ por cada oveja presente en la explotación al año $(48,8 \pm 5,91 \mathrm{kWh} /$ oveja en producción y año, o $25,8 \pm 2,39 \mathrm{kWh} /$ oveja presente en la explotación y año). Si expresamos el índice de utilización de la energía en función de la producción de leche, obtenemos un valor de $0,08 \pm 0,01 \mathrm{kWh}$ por cada litro de leche producido al día, es decir unos $84 \mathrm{kWh}$ por cada $1000 \mathrm{~L}$ de leche producida. Este valor es muy superior al observado por Bartolomé et al. (2013) en auditorías de consumo de electricidad en granjas de vacuno lechero (51 kWh/1000 L de leche). El consumo men- sual de electricidad (en $\mathrm{kWh} / \mathrm{mes}$ ) es mayor en las explotaciones medianas y grandes que en las pequeñas $(p<0,05)$; esto es consecuencia, fundamentalmente de la electricidad consumida en las labores de ordeño, que sigue un patrón similar $(\mathrm{p}<0,001 ;$ tabla III) con una media general de $37,1 \pm 3,18 \mathrm{kWh} /$ día. Sin embargo, estas diferencias dejan de ser significativas cuando el gasto se expresa en relación con el número de animales en producción o el número de animales presentes en la explotación. Cuando se calcula el consumo en relación con la cantidad de leche producida al día, se observa una tendencia $(p<0,10)$ de las explotaciones medianas a mostrar la mayor eficiencia, seguidas de las grandes, mientras que las pequeñas tienen los mayores consumos por litro de leche producida (tabla III). Por otra parte, cuando se realiza el cálculo del coste de la electricidad por animal presente en la explotación, éste tiende a ser mayor en las explotaciones más pequeñas y menor en las más grandes $(p<0,10)$, a consecuencia de las

Tabla III. Consumo de electricidad en las granjas de ganado ovino lechero de Castilla y León en función de su tamaño y de la raza explotada. (Electricity consumption in the dairy sheep farms of Castilla y León in terms of size and breed raised).

\begin{tabular}{|c|c|c|c|c|c|c|c|c|}
\hline & \multicolumn{4}{|c|}{ Tamaño } & \multicolumn{4}{|c|}{ Raza } \\
\hline & $\begin{array}{c}\text { pequeñas } \\
(n=12)\end{array}$ & $\begin{array}{c}\text { medianas } \\
(n=14)\end{array}$ & $\begin{array}{c}\text { grandes } \\
(n=9)\end{array}$ & $p$ & $\begin{array}{l}\text { Assaf } \\
(n=23)\end{array}$ & $\begin{array}{l}\text { Otras } \\
(n=12)\end{array}$ & $p$ & der \\
\hline \multicolumn{9}{|l|}{ Consumo (kWh) } \\
\hline /mes & $1023^{a}$ & $1981^{b}$ & $2643^{b}$ & ** & 1910 & 1936 & ns & 930,3 \\
\hline /oveja en producción/año & 67,3 & 40,7 & 42,9 & ns & 48,8 & 47,4 & ns & 31,37 \\
\hline /oveja presente/año & 31,8 & 26,3 & 20,4 & ns & 26,4 & 24,7 & ns & 12,85 \\
\hline /L de leche/día & $0,123^{b}$ & $0,062^{\mathrm{a}}$ & $0,076^{\mathrm{ab}}$ & $\mathrm{t}$ & 0,082 & 0,087 & ns & 0,0537 \\
\hline labores de ordeño/día & $20,2^{\mathrm{a}}$ & $39,8^{b}$ & $49,8^{b}$ & 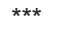 & 37,6 & 35,1 & ns & 14,10 \\
\hline \multicolumn{9}{|l|}{ Gastos (euros) } \\
\hline /mes & $204^{\mathrm{a}}$ & $378^{b}$ & $476^{b}$ & $* *$ & 368 & 347 & ns & 151,2 \\
\hline /oveja en producción/año & 13,5 & 7,8 & 7,7 & ns & 9,4 & 8,9 & ns & 5,78 \\
\hline /oveja presente/año & $6,4^{b}$ & $5,0^{\mathrm{ab}}$ & $3,6^{\mathrm{a}}$ & $\mathrm{t}$ & 5,1 & 4,7 & ns & 2,33 \\
\hline céntimos/L/leche/día & $2,56^{b}$ & $1,19^{a}$ & $1,40^{\mathrm{a}}$ & * & 1,59 & 1,75 & ns & 1,113 \\
\hline Energía reactiva ${ }^{1}(\%)$ & 4,3 & 4,1 & 5,2 & ns & 4,0 & 6,2 & ns & 2,20 \\
\hline
\end{tabular}

$n s=p>0,05 ; t=p<0,10 ;{ }^{*} p<0,05 ;{ }^{* *} p<0,01 ;{ }^{* * *} p<0,001 ;$ der $=$ desviación estándar residual.

${ }^{1}$ Generada por los equipos de la propia explotación y no consumida. 
diferencias tanto en el consumo como en el censo de animales en cada explotación. De forma general, el consumo de electricidad supone 367 euros al mes, 9,2 euros por oveja en producción y año o 4,9 euros por oveja presente en la explotación y año.

La repercusión que el gasto de electricidad supone por cada litro de leche producido al día es significativamente mayor $(\mathrm{p}<0,05)$ en las explotaciones más pequeñas $(0,026$ euros/L) que en las medianas y las grandes $(0,012$ y 0,014 euros/L, respectivamente), situándose el valor medio en torno a 0,016 euros/L de leche producida.

Esto da una idea de la gran variación que existe en función del tipo de explotación. Si bien se ofrecen los datos medios para todas las explotaciones encuestadas, es evidente la diferencia entre las grandes y las pequeñas. Es importante resaltar, por la trascendencia que supone en los costes de producción, que la repercusión económica del consumo de electricidad sobre la leche producida es mucho mayor en las explotaciones pequeñas.

Por lo que respecta a la distribución en las labores realizadas diariamente, no se observaron diferencias significativas debidas al tamaño de la granja ( $p>0,10)$ o a la raza explotada $(p>0,10)$, por lo que para simplificar la figura 1, sólo se muestra el valor de la media general. El ordeño (bomba de vacío + bomba de leche) consume alrededor de 34 kWh al día, más de un $40 \%$ del gasto eléctrico diario. El resto (figura 1), se destina fundamentalmente al enfriamiento de la leche (aproximadamente un $30 \%$ del consumo energético diario), el calentamiento del agua (alrededor del 12\% del consumo), el manejo de la alimentación y del estiércol y la iluminación. Con respecto a este último apartado, la mayoría de las lámparas en las explotaciones (casi el $80 \%$ ) son de tipo fluorescente, que tienen un bajo consumo eléctrico, si bien los focos halógenos representan un $14 \%$ del total de lámparas presentes y son utilizados, fundamentalmente, para la iluminación en el exterior.
En un estudio similar realizado en explotaciones de ganado vacuno lechero (Bartolomé et al., 2013), la distribución del consumo fue similar, salvando las distancias y pese a las particularidades y características intrínsecas a cada sistema de producción. Así, en las explotaciones de vacuno o de ovino lechero, la bomba de vacío es el equipo que presenta un mayor consumo, seguido del tanque de frío. En las granjas de vacuno, es más frecuente la presencia de otros equipos, como cintas de alimentación, sistemas de manejo del estiércol o arrastraderas hacen que los equipos clasificados bajo el epígrafe de otros lleven asociados un mayor porcentaje de consumo que en el caso de explotaciones de ovino.

Por lo que respecta a la implantación de medidas de ahorro energético en las explotaciones, ninguna de las auditadas posee sistema de preenfriamiento para la leche, mientras que 11 (un $31 \%$ ) tiene un variador de velocidad instalado en la bomba de vacío. La instalación de este tipo de dispositivos adecua el ritmo de trabajo de la bomba a la necesidad real en cada momento, por lo que se evita un consumo eléctrico innecesario, disminuyendo la sobrecarga del equipo y alargando su vida útil. Además, se produce una considerable disminución del ruido, con lo que se mejoran tanto las condiciones de trabajo de los operarios como el bienestar de los animales. En este sentido,

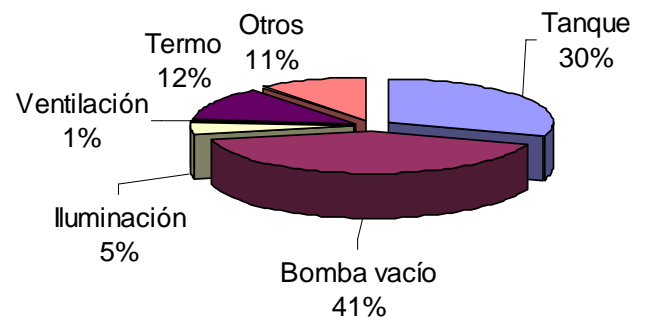

Figura 1. Distribución del consumo eléctrico medio entre los diferentes equipos en las granjas de ganado ovino lechero de Castilla y León. (Proportion of energy use among different equipment in dairy sheep farms in Castilla y León). 
las explotaciones que poseían variador de velocidad en la bomba de vacío presentaron un menor consumo por animal en producción que aquellas sin variador de velocidad ( 51 frente a $43 \mathrm{kWh} /$ oveja), si bien el consumo mensual fue mayor en las que sí presentaron variador de velocidad (2006 frente a $1909 \mathrm{kWh} / \mathrm{mes}$ ). El mayor número de animales en ordeño en aquellas explotaciones que sí tenían variador frente a las que no lo tenían ( 605 frente a 485 ) ha podido determinar el aumento en el consumo mensual de electricidad, mientras que la instalación del variador de velocidad, así como la configuración de la sala de ordeño, ha podido favorecer una mayor eficiencia en la utilización de la energía eléctrica por cada animal presente en la explotación.

En la tabla II aparece el coste que supone la electricidad (a precio de 2011), que se sitúa en torno a los 367 euros al mes, lo cual supondría una repercusión de alrededor de 1,6 céntimos de euro por cada litro de leche producido, es decir, un 2,2\% del precio por litro percibido por el ganadero (81,63 céntimos por litro, precio medio en 2011, MAGRAMA, 2012). En este sentido, todos los esfuerzos realizados para tratar de optimizar la utilización de la energía eléctrica, conllevarán una reducción de su repercusión en el precio de la leche. Lo cual, se traducirá en un incremento de los beneficios generados por la explotación.

Conviene no olvidar que parte del gasto en electricidad (alrededor de un $4 \%$ del consumo eléctrico que aparece en la factura) se corresponde con energía reactiva, generada por los equipos de la propia explotación y no consumida, lo que supone una penalización en la factura eléctrica. Este es un aspecto que hay que tener en cuenta a la hora de diseñar la instalación eléctrica de la explotación y tratar de adecuarla a la potencia que se vaya a consumir. El gasto en energía reactiva puede reducirse con la instalación de baterías de condensadores, que permitan un aprovechamiento de la misma y eviten la penalización por parte de la empre- sa suministradora de electricidad.

La realización de auditorías energéticas es imprescindible para conocer de manera fiable cómo se produce el consumo eléctrico en una explotación y cuáles son los factores que afectan al mismo. Esta información, junto con la relativa a la productividad de la granja permitirá calcular los índices de utilización de la energía, así como evaluar las distintas oportunidades de ahorro en función de su rentabilidad económica (IDAE, 2010). El objetivo final será el establecimiento de medidas de ahorro y prácticas de manejo que permitan realizar un uso más racional de la energía, promover el ahorro e incrementar la eficiencia energética de las explotaciones. Todo ello con la finalidad de llevar a cabo la actividad ganadera dentro de un marco de sostenibilidad económica y ambiental.

\section{CONCLUSIONES}

El ordeño supone más del $40 \%$ del consumo total de electricidad de una explotación de ovino lechero y tanto la eficiencia del ordeño como la eficiencia en la utilización de la energía parecen depender del tamaño de las explotaciones. El consumo total de electricidad representa el $2 \%$ del precio percibido por cada litro de leche vendido. La implementación de medidas de ahorro (variadores y sistemas de enfriamiento e iluminación eficientes) permitiría reducir el consumo energético de la producción de leche, minimizar su impacto ambiental y aumentar su productividad (menor repercusión económica sobre el precio de la leche).

\section{AGRADECIMIENTOS}

Este trabajo ha sido financiado por el proyecto DER20111800060100199 de la Red Rural Nacional, Ministerio de Medio Ambiente y Medio Rural y Marino (MARM) y por el Fondo Europeo Agrícola de Desarrollo Rural (FEADER): Europa invierte en las zonas rurales. 


\section{BODAS ETAL.}

\section{BIBLIOGRAFÍA}

Bartolomé, D.J.; Posado, R.; Bodas, R.; Tabernero de Paz, M.J.; García, J.J. y Olmedo, S. 2013. Caracterización del consumo eléctrico en las granjas de vacuno lechero de Castilla y León. Arch Zootec, 62: 447-455.

Castañares, N. 2008. Recría de corderas de raza Assaf Española. Efecto de la alimentación entre el nacimiento y los cinco meses de edad sobre el crecimiento de los animales, el desarrollo de la glándula mamaria y la producción de leche en la primera lactación. Tesis Doctoral. Universidad de León. 251 pp.

Edens, W.C.; Pordesimo, L.O.; Wilhelm, L.R. and Burns, R.T. 2003. Energy use analysis of major milking center components at a dairy experiment station. Appl Eng Agric, 19: 711-716.

IDAE. 2010. Auditorías energéticas en instalaciones ganaderas. Parte 1. Manual para la realización de auditorías energéticas en instalaciones ganaderas. Serie: Ahorro y eficiencia energética en la agricultura. Instituto para la diversificación y ahorro de la energía. Madrid. 83 pp.

INE. 2002. Censo agrario 1999. Instituto Nacional de Estadística. http://www.ine.es/inebmenu/ mnu_agricultura.htm (28/05/2012).

INE. 2012. Censo agrario 2009. Instituto Nacional de Estadística. http://www.ine.es/inebmenu/ mnu_agricultura.htm (28/05/2012).

Lavín, P.; Mantecón, A.R. y Giradles, F.J. 1997. Análisis productivo-económico de las explotaciones ovinas de leche basadas en las razas Churra y Assaf. XXIX Jornadas de Estudio AIDA. ITEA Vol. Extra 18 (II): 782-785.

Ludington, D. and Johnson, E.L. 2003. Dairy farm energy audit summary report. New York State Energy Research and Development Authority. http://www.nyserda.ny.gov/ /media/Files/
Publications/Energy\%20Audit\%20Reports/ dairy-farm-energy.ashx (22/05/2012). 25 pp.

MARM. 2011. Anuario de Estadística Agraria y Agroalimentaria. Ministerio de Medio Ambiente y Medio Rural y Marino. http://www.magrama. gob.es/estadistica/pags/anuario/2010/ae 2010_avance.pdf (03/05/2012). 1219 pp.

MAGRAMA. 2012. Publicación de indicadores de precios y salarios agrarios. Ministerio de Agricultura, Alimentación y Medio Ambiente. http:// www.magrama.gob.es/es/estadistica/temas/ estadisticas-agrarias/economia/preciospercibidos-pagados-salarios/publicacion-deindicadores-de-precios-y-salarios-agrarios/ default.aspx (12/06/2012). 17 pp.

Murgia, L.; Caria, M. and Pazzona, A. 2008. Energy use and management in dairy farms. In: Innovation technology to empower safety, health and welfare in agriculture and agro-food systems. International conference. September 15-17. Ragusa-Italy. http://www.ragusashwa. it/CD_2008/lavori/TOPIC9/orale/MURGIA.pdf (22/05/2012).

Rodríguez, L.; Martínez, F.; Herrera, E.; Sopena, J.; Real, M.A.; García, I.; García, M.; Matilla, J.; González, M.A.; Padrones, J.; Gamarra, A. y García, J.I. 2003. Gestión técnico económica en explotaciones de Ovino de Castilla y León. Aspectos destacables: campañas 1998-2002 XXVIII Jornadas Científicas y VII Jornadas Internacionales de la Sociedad Española de Ovinoctenia y Caprinotecnia. Badajoz. pp. 412414.

Ugarte, E.; Ruiz, R.; Gabiña, D. and Beltrán de Heredia, I. 2001. Impact of high-yielding foreign breeds on the Spanish dairy sheep industry. Livest Prod Sci, 71: 3-10. 